\title{
The Infections of Lower Genital Tract
}

\author{
Francesco De Seta, ${ }^{1}$ Secondo Guaschino, ${ }^{2}$ Bryan Larsen, ${ }^{3}$ \\ Gilbert Donders, ${ }^{4}$ and Gonzaga Andabati ${ }^{5}$
}

\author{
${ }^{1}$ Department of Reproductive and Developmental Sciences, IRCCS Burlo Garofolo, University of Trieste, 34100 Trieste, Italy \\ ${ }^{2}$ Department of Obstetrics and Gynecology, Institute for Maternal and Child Health, IRCCS Burlo Garofolo, University of Trieste, \\ Via dell'Istria, 34100 Trieste, Italy \\ ${ }^{3}$ College of Osteopathic Medicine, Marian University, Indianapolis, IN 46222, USA \\ ${ }^{4}$ Department of Obstetrics and Gynecology, Regional Hospital H Hart Tienen, University Hospital Gasthuisberg Leuven, \\ and University Hospital Citadelle Liége, Liége, Belgium \\ ${ }^{5}$ Department of Obstetrics and Gynaecology, College of Health Sciences, Makerere University, P.O. Box 7072, Kampala, Uganda
}

Correspondence should be addressed to Francesco De Seta, fradeseta@gmail.com and Bryan Larsen, blarsen@marian.edu

Received 23 October 2012; Accepted 23 October 2012

Copyright ( 2012 Francesco De Seta et al. This is an open access article distributed under the Creative Commons Attribution License, which permits unrestricted use, distribution, and reproduction in any medium, provided the original work is properly cited.

Infections occurring in the lower female genital tract represent a complex topic from several different viewpoints. First, there is a body of scientific knowledge and clinical consequences associated with these infections. Second, the complexity is increased by the biological diversity of infectious organisms involved including bacteria, fungi, virus, and protozoan life forms. A third layer of complexity exists in the interaction between the host and infectious organisms through the inflammatory responses to infections and through the elaboration of host factors such as secreted antibodies and defensin molecules inhibitory to intravaginal microorganisms. Finally, the vagina is host to an indigenous microbiota which is credited with contributing to vaginal health, but also the flora interacts with exogenous microorganisms involved in pathogenesis of vaginal infections.

It is striking that despite the fact that the vaginal flora has been a topic of interest to physicians and scientists since the last decade of the 19th century, there is still much to be learned and newer research techniques are continuing to elucidate our understanding of the vaginal flora, infectious agents involved in pathogenic interactions with the host, and the effects of indigenous and exogenous organisms on the physiology of the vagina. Because of continuing discoveries, the offering of the present special issue is an appropriate venue for presentation of current thinking about vaginal infections. The editors are pleased to present the articles published in this issue.
As mentioned above, the vaginal infections derive from a biologically diverse collection of microbes. Thus, the reader will find that this organisms represented in this issue include bacteria (Group B Streptococcus in the A. Lambiase et al. article, Treponema pallidum in the M. de Santis et al. contribution, and Lactobacilli and bacterial vaginosis associated bacteria in the works of J. M. Bohbot and J. M. Cardot, C. Mitchell et al.). Viral pathogens are represented by Herpes Simplex in the G. Straface et al. article, HIV in the C. Vallone et al. contribution, and HPV in the M. Guadalupe et al. paper. Candida species are addressed by A. Palmeira-deOliviera and coworkers, while the continuum of biological forms is rounded out by the R. Sehgal et al. paper which focuses on Trichomonas.

Another aspect of vaginal infections is the diversity and disparity of epidemiologic details depending on both geography and ethnicity of the populations studied. Several articles in this issue provide information about populations derived from various locations across the globe. It is always useful to become informed about common issues along with differences in prevalence and manifestations at locations other than one's own practice site.

In addition to efforts of physicians and scientists to define and characterize vaginal infections in terms of microbiology and pathophysiology, there is always the overarching question of how we can best deal with these clinical conditions through available or new therapies. The prevailing logic 
of using antibiotics to deal with these issues has been both logically and widely applied since the discovery of penicillin in the middle of the last century. But as indicated by $M$. de Santis et al. review that despite highly effective penicillin applied to treating syphilis, the disease has not been eradicated and congenital syphilis in particular remains a continuing problem. Certainly history has reminded us that the ability of microorganisms to develop resistance to antimicrobial drugs has altered are ability to treat some infections. A. Lambiase and coworkers have explored the occurrence of resistance to macrolides and clindamycin among GBS isolates in Italy. Likewise antiviral drugs may interfere with neonatal infection with Herpes Simplex during pregnancy (G. Straface et al.). In addition to traditional antibiotics, investigators are continuing to discover unanticipated antimicrobial attributes of drugs as illustrated by the work of A. Palmeria-de-Oliviera et al. who studied the anti-Candida activity of nitroglycerine and lidocaine. As with any compound with antimicrobial activity in vitro, the translation of the initial findings to clinical use will be a continuing challenge.

Knowing that antibiotics, despite phenomenal historical successes, have not been able to provide a full and final answer to vaginal infections, investigators have turned their attention to exploiting host defense mechanisms in a variety of ways and employing various mechanisms of action. For example, based on the concept that normal flora organisms promote vaginal health, the use of probiotic organisms continues to be studied in relation to bacterial vaginosis (see J. M. Bohbot and J. M. Cardot). In addition to probiotics, known host defense factors have been studied as potential therapeutic drugs. A review of the possible value of lactoferrin as a therapy for the very prevalent Trichomonas vaginalis was examined by $\mathrm{R}$. Sehgal and coworkers.

This issue should serve as a reminder that many questions remain about the content of the healthy vagina and the specific organisms capable of producing symptomatic infection. The challenge for the future is to continue to examine both the science behind vulvovagianl infectious pathology and to devise clinically relevant therapeutic approaches to the problems that continue to make women uncomfortable, undermine the quality of life, and in some cases threaten their overall health and the health of their fetuses and infants. We owe it to our wives, mothers, and daughters to continue to bring our intellectual efforts to bear on developing creative approaches to understanding and addressing vaginal infections of all types and in all populations no matter where they are in the world. 


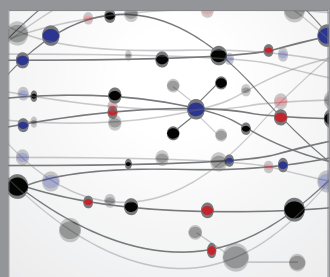

The Scientific World Journal
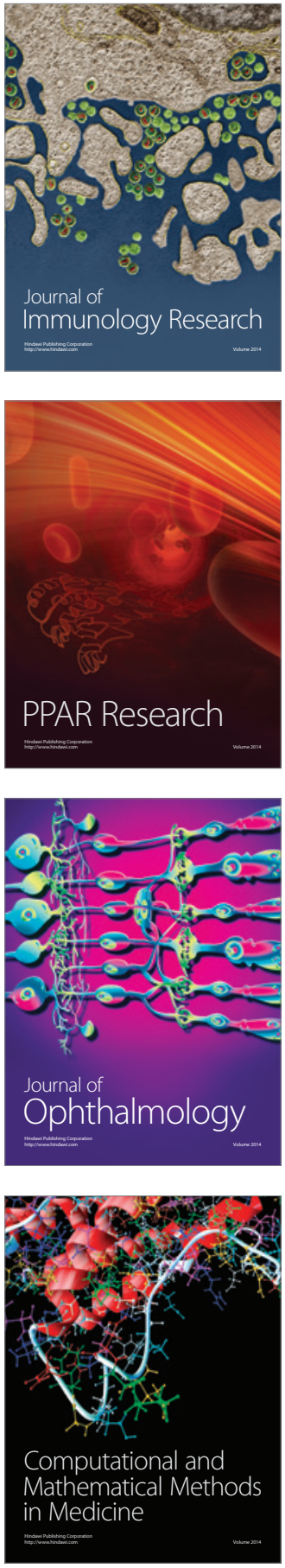

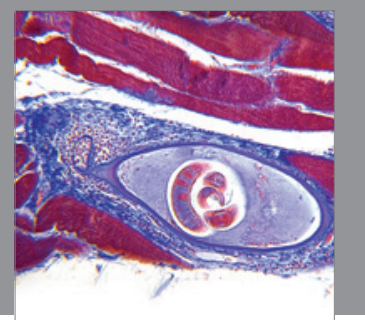

Gastroenterology

Research and Practice
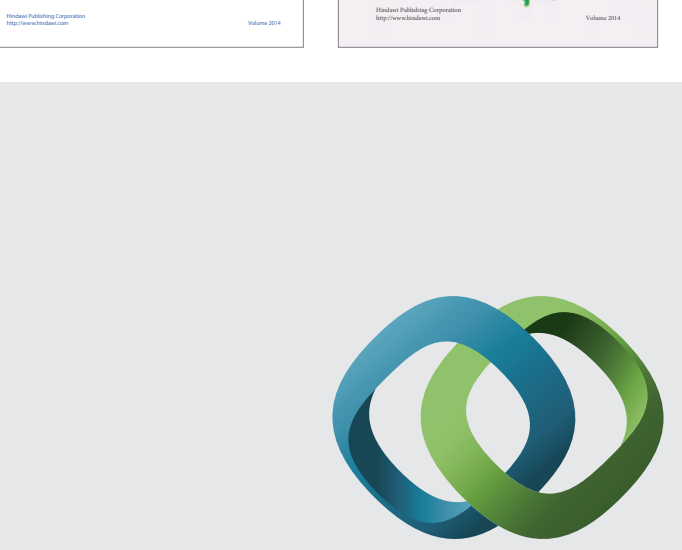

\section{Hindawi}

Submit your manuscripts at

http://www.hindawi.com
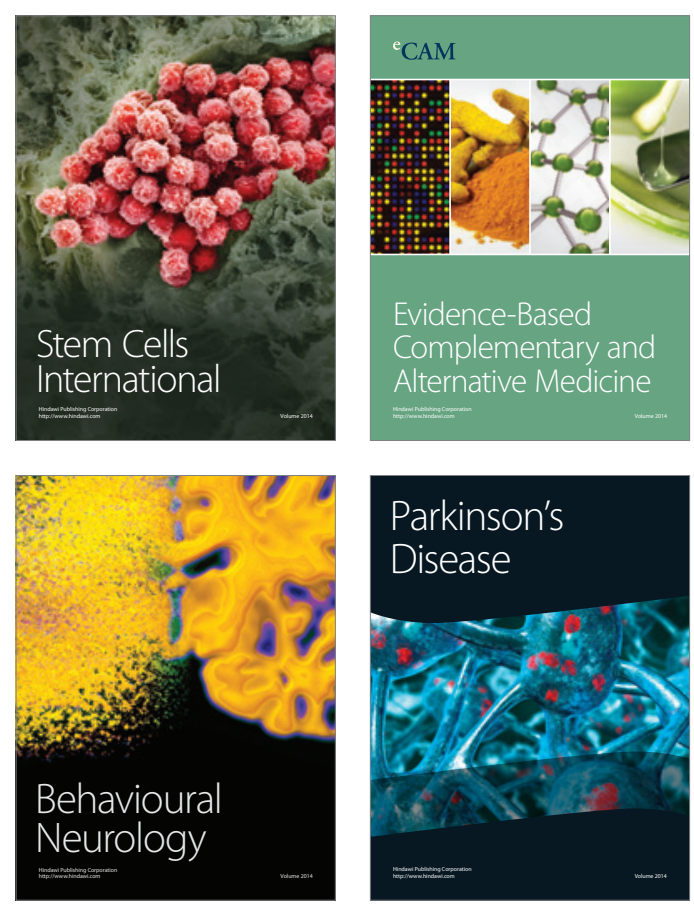

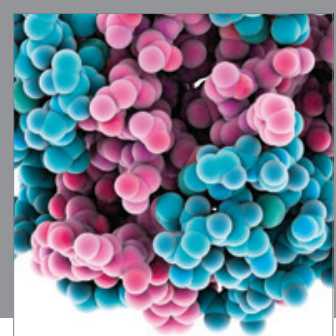

Journal of
Diabetes Research

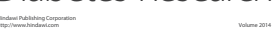

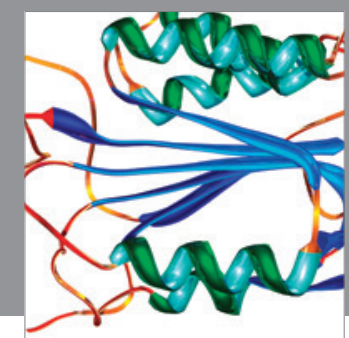

Disease Markers
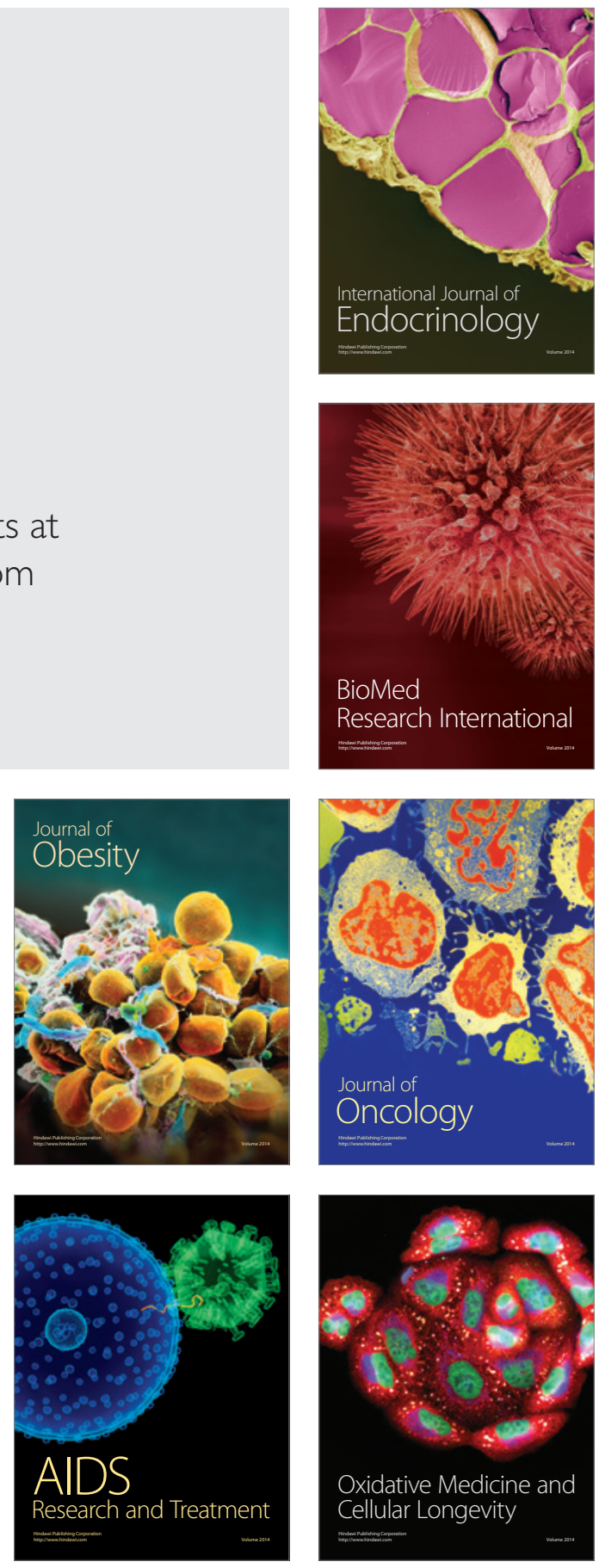